\title{
Natural orbitals of the ground state of the two-electron harmonium atom
}

\author{
Jerzy Cioslowski ${ }^{1,2}$
}

Received: 19 April 2018 / Accepted: 6 June 2018 / Published online: 11 November 2018

(c) The Author(s) 2018

\begin{abstract}
The radial components of the natural orbitals (NOs) pertaining to the ${ }^{1} S_{+}$ground state of the two-electron harmonium atom are found to satisfy homogeneous differential equations at the values of the confinement strength $\omega$ at which the respective correlation factors are given by polynomials. Together with the angular momentum $l$ of the NOs, the degrees of these polynomials determine the orders of the differential equations, eigenvalues of which (arising from well-defined boundary conditions) yield the natural amplitudes. In the case of $l=0$, analysis of these equations uncovers certain properties of the NOs whereas application of a WKB-like approximation produces asymptotic expressions for both the NOs and the corresponding natural amplitudes that hold when the latter are small negative numbers. Extensive numerical calculations reveal that these expressions remain valid for arbitrary values of $\omega$. The approximate $s$-type NOs, which are remarkably accurate at sufficiently small radial distances and exhibit universal scaling, differ qualitatively from the eigenfunctions of the core Hamiltonian even at the $\omega \rightarrow \infty$ limit of vanishing electron correlation.
\end{abstract}

Keywords Two-electron harmonium atom $\cdot$ Natural orbitals $\cdot$ WKB approximation $\cdot$ Hill's asymptotics

\section{Introduction}

The time-independent Schrödinger equation with the Hamiltonian

$\hat{H}=-\frac{1}{2}\left(\hat{\nabla}_{1}^{2}+\hat{\nabla}_{2}^{2}\right)+\frac{1}{2} \omega^{2}\left(r_{1}^{2}+r_{2}^{2}\right)+\frac{1}{r_{12}}$

possesses closed-form solutions for infinitely many values of the confinement strength $\omega$ [1]. For this reason, the nonrelativistic system it describes, known as the two-electron harmonium atom, is often employed as a benchmarking tool in testing of approximate electronic structure methods, including those based on the density functional theory [2-12] and other formalisms [13-16]. Thanks to realistic electron

Published as part of the special collection of articles In Memoriam of János Àngyán.

Jerzy Cioslowski

jerzy@wmf.univ.szczecin.pl

1 Institute of Physics, University of Szczecin, Wielkopolska 15, 70-451 Szczecin, Poland

2 Max-Planck-Institut für Physik komplexer Systeme, Nöthnitzer Str. 38, D-01187 Dresden, Germany densities involved, such tests are more suitable for calibration of density functionals and assessment of their accuracy than those relying on the homogeneous electron gas.

Electronic properties of the two-electron harmonium atom are readily elucidated not only at select values of $\omega$ but also at the weak- and strong-correlation limits. Within the former regime, which corresponds to strong confinement, the Hamiltonian (1) describes a system of two weakly coupled three-dimensional harmonic oscillators that is amenable to perturbative treatment [17-22]. On the other hand, the strongly correlated species that ensues at small values of $\omega$ can be regarded as a classical Wigner molecule subject to minor quantum corrections [17, 23].

In this paper, some recently derived properties of natural orbitals (NOs) and their occupation numbers pertaining to the ${ }^{1} S_{+}$ground state of the two-electron harmonium atom are reported. Although this state has been the subject of numerous studies involving both rigorous mathematical analysis $[1,17-30]$ and numerical approaches [17, 28], the attention devoted to the corresponding NOs has been limited to investigations of their asymptotic behavior at the $\omega \rightarrow 0$ limit [23] and of their collective occupancies at various values of $\omega$ [24-26], formulation of accurate approximations to the strongly occupied NOs at $\omega=\frac{1}{2}$ and $\omega=\frac{1}{10}$ [27], and finding definitive answers [28] to the questions concerning the 
existence of NOs with vanishing occupation numbers [17, $29,30]$. With amelioration of this unsatisfactory state of knowledge as its objective, the present work aims at uncovering universalities in NOs and their occupation numbers that persist throughout the entire range of confinement strengths.

\section{Theory}

The spatial part $\Psi\left(\vec{r}_{1}, \vec{r}_{2}\right) \equiv \Psi\left(\omega ; \vec{r}_{1}, \vec{r}_{2}\right)$ of the ${ }^{1} S_{+}$groundstate wavefunction of the two-electron harmonium atom reads $[1,17]$

$\Psi\left(\vec{r}_{1}, \vec{r}_{2}\right)=\exp \left[-\frac{\omega}{2}\left(r_{1}^{2}+r_{2}^{2}\right)\right] g\left(r_{12}\right)$,

where the correlation factor $g\left(r_{12}\right) \equiv g\left(\omega ; r_{12}\right)$ (inclusive of the normalization constant) is given by the polynomials

$g\left(\omega_{k} ; r\right)=\sum_{j=0}^{k} C_{j}\left(\omega_{k}\right) r^{j}$

for certain values of $\omega \in\left\{\omega_{k}\right\}(k \geq 1)$, the first four elements of the set $\left\{\omega_{k}\right\}$ being $\omega_{1}=\frac{1}{2}, \omega_{2}=\frac{1}{10}, \omega_{3}=\frac{5-\sqrt{17}}{24}$, and $\omega_{4}=\frac{35-3 \sqrt{57}}{712}[1,17]$. It is worth noting that, since the ratio $C_{1}(\omega) / C_{0}(\omega)$ is fixed at $\frac{1}{2}$ by the electron-coalescence cusp condition, setting $\vec{r}_{1}=\vec{r}_{2}=0$ in Eq. (2) yields $C_{0}\left(\omega_{k}\right)=\Psi\left(\omega_{k} ; \overrightarrow{0}, \overrightarrow{0}\right)$ and $C_{1}\left(\omega_{k}\right)=\frac{1}{2} \Psi\left(\omega_{k} ; \overrightarrow{0}, \overrightarrow{0}\right)$. Throughout the text, the $\{\operatorname{Re}(\Psi(\overrightarrow{0}, \overrightarrow{0}))>0, \operatorname{Im}(\Psi(\overrightarrow{0}, \overrightarrow{0}))=0\}$ phase convention is employed for the wavefunction, which is assumed to be square-normalized to one.

The spatial parts $\left\{\psi_{n l m}(\vec{r})\right\} \equiv\left\{\psi_{n l m}(\omega ; \vec{r})\right\}$ of the NOs pertaining to $\Psi\left(\vec{r}_{1}, \vec{r}_{2}\right)$ are eigenfunctions of a homogeneous Fredholm equation of the second kind [31]

$\int \Psi\left(\vec{r}_{1}, \vec{r}_{2}\right) \psi_{n l m}\left(\vec{r}_{2}\right) \mathrm{d} \vec{r}_{2}=\lambda_{n l} \psi_{n l m}\left(\vec{r}_{1}\right)$.

Squares of absolute values of the respective natural amplitudes $\left\{\lambda_{n l}\right\} \equiv\left\{\lambda_{n l}(\omega)\right\}$ equal the occupation numbers $\left\{v_{n l}\right\} \equiv\left\{v_{n l}(\omega)\right\}$. Thanks to the spherical symmetry of the underlying wavefunction, the angular degrees of freedom can be integrated out from Eq. (4), yielding [compare Eq. (2)]

$$
\begin{aligned}
& \frac{4 \pi}{2 l+1} \int_{0}^{\infty} \exp \left[-\frac{\omega}{2}\left(r_{1}^{2}+r_{2}^{2}\right)\right] g_{l}\left(r_{1}, r_{2}\right) \phi_{n l}\left(r_{2}\right) r_{2}^{2} \mathrm{~d} r_{2} \\
& \quad=\lambda_{n l} \phi_{n l}\left(r_{1}\right)
\end{aligned}
$$

for the NOs with the angular momenta of $l[27,30]$. In Eq. (5), $\left\{\phi_{n l}(r)\right\} \equiv\left\{\phi_{n l}(\omega ; r)\right\}$ (normalized according to $\left.\int_{0}^{\infty}\left[\phi_{n l}(r)\right]^{2} r^{2} \mathrm{~d} r=1\right)$ are the real-valued radial parts of $\left\{\psi_{n l m}(\vec{r})\right\}$,

$\psi_{n l m}(\vec{r})=\phi_{n l}(r) Y_{l}^{m}(\theta, \varphi)$,

and the angularly averaged correlation factor $g_{l}\left(r_{1}, r_{2}\right) \equiv$ $g_{l}\left(\omega ; r_{1}, r_{2}\right)$ reads

$$
\begin{aligned}
g_{l}\left(r_{1}, r_{2}\right)= & \frac{2 l+1}{2 r_{1} r_{2}} \\
& \times \int_{\left|r_{1}-r_{2}\right|}^{r_{1}+r_{2}} P_{l}\left(\frac{r_{1}^{2}+r_{2}^{2}-r_{12}^{2}}{2 r_{1} r_{2}}\right) g\left(r_{12}\right) r_{12} \mathrm{~d} r_{12},
\end{aligned}
$$

where the standard notation of $Y_{l}^{m}(\theta, \varphi)$ and $P_{l}(t)$ is used for the respective spherical harmonic and Legendre polynomial.

\subsection{A differential equation for NOs}

Combining Eqs. (3), (5), and (7) affords

$$
\begin{aligned}
& 2 \pi \int_{0}^{r_{1}} \exp \left(-\omega_{k} r_{2}^{2}\right)\left[\sum_{j=0}^{k} C_{k j} U_{l j}\left(r_{1}, r_{2}\right)\right] \chi_{k n l}\left(r_{2}\right) \mathrm{d} r_{2} \\
& +2 \pi \int_{0}^{\infty} \exp \left(-\omega_{k} r_{2}^{2}\right)\left[\sum_{j=0}^{k} C_{k j} W_{l j}\left(r_{1}, r_{2}\right)\right] \chi_{k n l}\left(r_{2}\right) \mathrm{d} r_{2} \\
& \quad=\lambda_{k n l} r_{1}^{2 l} \chi_{k n l}\left(r_{1}\right),
\end{aligned}
$$

where the eigenfunctions $\left\{\chi_{k n l}(r)\right\} \equiv\left\{\chi_{n l}\left(\omega_{k} ; r\right)\right\}$ are related to the spatial parts of NOs through the equation

$\chi_{k n l}(r)=\exp \left(\frac{\omega_{k}}{2} r^{2}\right) r^{1-l} \phi_{n l}\left(\omega_{k} ; r\right)$.

Here and in the following, the notation $C_{k j} \equiv C_{j}\left(\omega_{k}\right)$ and $\lambda_{k n l} \equiv \lambda_{n l}\left(\omega_{k}\right)$ is employed for the sake of convenience, whereas the standard notation of $P_{l}^{(q)}(t)$ is used for the $q$ th derivative of the respective Legendre polynomial in the definitions

$$
\begin{aligned}
& U_{l j}\left(r_{1}, r_{2}\right)=\left[(-1)^{j}-1\right] \sum_{q=0}^{l} \frac{\left(-\frac{1}{2}\right)^{q}\left(r_{1} r_{2}\right)^{l-q}}{(j+2 q+2) q !} \\
& \quad \times P_{l}^{(q)}\left(\frac{r_{1}^{2}+r_{2}^{2}}{2 r_{1} r_{2}}\right)\left(r_{1}-r_{2}\right)^{j+2 q+2}
\end{aligned}
$$

and

$$
\begin{aligned}
& W_{l j}\left(r_{1}, r_{2}\right)=\sum_{q=0}^{l} \frac{\left(-\frac{1}{2}\right)^{q}\left(r_{1} r_{2}\right)^{l-q}}{(j+2 q+2) q !} \\
& \quad \times P_{l}^{(q)}\left(\frac{r_{1}^{2}+r_{2}^{2}}{2 r_{1} r_{2}}\right)\left[\left(r_{1}+r_{2}\right)^{j+2 q+2}-\left(r_{2}-r_{1}\right)^{j+2 q+2}\right] .
\end{aligned}
$$


As the sums over $j$ in the 1.h.s. of Eq. (8) are polynomials of degree $2 l+2\left[\frac{k+1}{2}\right]+1$ in $r_{1}$ (here and in the following, $[t]$ denotes the integer part of $t$ ), differentiating both sides of this equation $2 l+2\left[\frac{k+1}{2}\right]+2$ times with respect to $r_{1}$ produces a homogeneous differential equation for $\chi_{k n l}(r)$ that reads is the first member of the sequence of large- $r$ asymptotic approximants $\left\{\chi_{k n l}^{>[q]}\left(r_{1}\right)\right\}$ defined recursively as [compare Eq. (8)]

$2 \pi\left(\frac{\partial}{\partial r_{1}}\right)^{2 l+2\left[\frac{k+1}{2}\right]+2} \int_{0}^{r_{1}} \exp \left(-\omega_{k} r_{2}^{2}\right)\left[\sum_{j=0}^{k} C_{k j} U_{l j}\left(r_{1}, r_{2}\right)\right] \chi_{k n l}\left(r_{2}\right) \mathrm{d} r_{2}=\lambda_{k n l}\left(\frac{\partial}{\partial r_{1}}\right)^{2 l+2\left[\frac{k+1}{2}\right]+2}\left[r_{1}^{2 l} \chi_{k n l}\left(r_{1}\right)\right]$.

Together with $2 l+2\left[\frac{k+1}{2}\right]+2$ boundary conditions

$$
\begin{aligned}
& 2 \pi\left(\frac{\partial}{\partial r_{1}}\right)^{p}\left(r_{1}^{-2 l} \int_{0}^{r_{1}} \exp \left(-\omega_{k} r_{2}^{2}\right)\left[\sum_{j=0}^{k} C_{k j} U_{l j}\left(r_{1}, r_{2}\right)\right] \chi_{k n l}\left(r_{2}\right) \mathrm{d} r_{2}\right. \\
& \left.+r_{1}^{-2 l} \int_{0}^{\infty} \exp \left(-\omega_{k} r_{2}^{2}\right)\left[\sum_{j=0}^{k} C_{k j} W_{l j}\left(r_{1}, r_{2}\right)\right] \chi_{k n l}\left(r_{2}\right) \mathrm{d} r_{2}\right)\left.\right|_{r_{1}=0}=\lambda_{k n l} \chi_{k n l}^{(p)}(0), \quad p=0, \ldots, 2 l+2\left[\frac{k+1}{2}\right]+1,
\end{aligned}
$$

this eigenequation is fully equivalent to Eq. (8).

There are $l+\left[\frac{k+1}{2}\right]+1$ conditions (13) corresponding to even $p$ that simply imply vanishing of $\chi_{k n l}(r)$ and all its evenorder derivatives at $r=0$. The remaining $l+\left[\frac{k+1}{2}\right]+1$ conditions that ensue for odd $p$ are rather cumbersome. However, satisfying them is equivalent to enforcing the large- $r$ asymptotics of $\chi_{k n l}(r) \underset{r \rightarrow \infty}{\longrightarrow} \chi_{k n l}^{>}(r)+o(1)$, where the rational function

$$
\begin{aligned}
\chi_{k n l}^{>}\left(r_{1}\right)= & \frac{2 \pi}{\lambda_{k n l}} r_{1}^{-2 l} \int_{0}^{\infty} \exp \left(-\omega_{k} r_{2}^{2}\right) \\
& \times \sum_{j=0}^{k} C_{k j}\left[U_{l j}\left(r_{1}, r_{2}\right)+W_{l j}\left(r_{1}, r_{2}\right)\right] \chi_{k n l}\left(r_{2}\right) \mathrm{d} r_{2}
\end{aligned}
$$

$$
\begin{aligned}
& \chi_{k n l}^{>[q+1]}\left(r_{1}\right)=\chi_{k n l}^{>}\left(r_{1}\right)-\frac{2 \pi}{\lambda_{k n l}} r_{1}^{-2 l} \int_{r_{1}}^{\infty} \exp \left(-\omega_{k} r_{2}^{2}\right) \\
& \times \sum_{j=0}^{k} C_{k j} U_{l j}\left(r_{1}, r_{2}\right) \chi_{k n l}^{>[q]}\left(r_{2}\right) \mathrm{d} r_{2} .
\end{aligned}
$$

\subsection{The case of $I=0$}

For $l=0$, the differential Eq. (12) and the boundary conditions (13) assume particularly simple forms, namely

$-4 \pi \sum_{j=0}^{\left[\frac{k-1}{2}\right]}(2 j+2) ! C_{k, 2 j+1}\left(\frac{\partial}{\partial r_{1}}\right)^{2\left[\frac{k-1}{2}\right]-2 j}\left[\exp \left(-\omega_{k} r_{1}^{2}\right) \chi_{k n 0}\left(r_{1}\right)\right]=\lambda_{k n 0}\left(\frac{\partial}{\partial r_{1}}\right)^{2\left[\frac{k+3}{2}\right]} \chi_{k n 0}\left(r_{1}\right)$,

together with

$\chi_{k n 0}^{(p)}(0)=0, \quad p=0,2, \ldots, 2\left[\frac{k+1}{2}\right]$

and

$-\left.4 \pi \sum_{j=0}^{\min \left(\left[\frac{k-1}{2}\right], \frac{p-5}{2}\right)}(2 j+2) ! C_{k, 2 j+1}\left(\frac{\partial}{\partial r_{1}}\right)^{p-2 j-4}\left[\exp \left(-\omega_{k} r_{1}^{2}\right) \chi_{k n 0}\left(r_{1}\right)\right]\right|_{r_{1}=0}$

$+\sum_{j=\max (0, p-2)}^{k} \frac{(j+1) !}{(j+2-p) !} C_{k j} \mu_{k n 0, j+2-p}=\lambda_{k n 0} \chi_{k n 0}^{(p)}(0), \quad p=1,3, \ldots, 2\left[\frac{k+1}{2}\right]+1$, 
where

$\mu_{k n l, j}=4 \pi \int_{0}^{\infty} \exp \left(-\omega_{k} r^{2}\right) r^{j} \chi_{k n l}(r) \mathrm{d} r$.

The large- $r$ asymptotics becomes a polynomial (note the linear dependences among its coefficients)

$$
\begin{gathered}
\chi_{k n 0}^{>}(r)=\lambda_{k n 0}^{-1}\left(\sum_{j=1}^{\left[\frac{k+1}{2}\right]} \frac{1}{2 j+1} C_{k, 2 j-1} \mu_{k n 0,2 j+1}\right. \\
\left.+\sum_{p=1}^{k+1} \frac{r^{p}}{p !} \sum_{j=0}^{\left[\frac{k+1-p}{2}\right]} \frac{(2 j+p) !}{(2 j+1) !} C_{k, 2 j+p-1} \mu_{k n 0,2 j+1}\right)
\end{gathered}
$$

and the approximants (15) are given by

$$
\begin{aligned}
& \chi_{k n 0}^{>[q+1]}\left(r_{1}\right)=\chi_{k n 0}^{>}\left(r_{1}\right)+\frac{4 \pi}{\lambda_{k n 0}} \int_{r_{1}}^{\infty} \exp \left(-\omega_{k} r_{2}^{2}\right) \\
& \quad \times \sum_{j=0}^{\left[\frac{k-1}{2}\right]} \frac{C_{k, 2 j+1}}{2 j+3}\left(r_{1}-r_{2}\right)^{2 j+3} \chi_{k n 0}^{>[q]}\left(r_{2}\right) \mathrm{d} r_{2} .
\end{aligned}
$$

\subsection{The $s$-type NOs pertaining to $\omega=\omega_{1}$ and $\omega=\omega_{2}$}

For the first two instances of the explicitly known eigenfunctions of the Hamiltonian (1), the sum in the 1.h.s. of Eq. (16) reduces to a single term, yielding

$$
\begin{aligned}
-8 & \pi C_{k 1} \exp \left(-\omega_{k} r^{2}\right) \chi_{k n 0}(r)=\lambda_{k n 0} \chi_{k n 0}^{(4)}(r), \\
k=1,2 . &
\end{aligned}
$$

Despite arising from the same eigenequation, the eigenfunctions $\chi_{1 n 0}(r)$ and $\chi_{2 n 0}(r)$ are not related through an argument/ value scaling because of their different large- $r$ asymptotics, namely [compare Eq. (20)]

$\chi_{1 n 0}^{>}(r)=\lambda_{1 n 0}^{-1}\left(\frac{1}{3} C_{11} \mu_{1 n 0,3}+C_{10} \mu_{1 n 0,1} r+C_{11} \mu_{1 n 0,1} r^{2}\right)$

versus

$$
\begin{gathered}
\chi_{2 n 0}^{>}(r)=\lambda_{2 n 0}^{-1}\left(\frac{1}{3} C_{21} \mu_{2 n 0,3}+\left(C_{20} \mu_{2 n 0,1}+C_{22} \mu_{2 n 0,3}\right) r\right. \\
\left.+C_{21} \mu_{2 n 0,1} r^{2}+C_{22} \mu_{2 n 0,1} r^{3}\right) .
\end{gathered}
$$

It should be emphasized that none of the NOs can be unoccupied as setting $\lambda_{k n 0}=0$ in Eq. (22) would imply vanishing of $\chi_{k n 0}(r)$ for all $r$. On the other hand, integration of this eigenequation subject to the boundary conditions (17) produces

$$
\begin{aligned}
\chi_{k n 0}^{(2)}(r)=\chi_{k n 0}^{(3)}(0) r-8 \pi \lambda_{k n 0}^{-1} C_{k 1} \\
\quad \times \int_{0}^{r} \chi_{k n 0}\left(r^{\prime}\right) \exp \left(-\omega_{k} r^{\prime 2}\right)\left(r-r^{\prime}\right) \mathrm{d} r^{\prime},
\end{aligned}
$$

allowing for elucidation of some properties of the NOs. To achieve this goal, one conveniently assumes without any loss of generality that $\chi_{k n 0}^{(1)}(0)>0$ (i.e., the respective NO is positive-valued at $r=0$ ) and notes that $C_{k 1}>0$. Inspection of Eq. (25) leads to the conclusion that the combination of $\chi_{k n 0}^{(3)}(0)>0$ and $\lambda_{k n 0}<0$ assures $\chi_{k n 0}^{(2)}(r)$ being positive-valued for $r>0$ and thus $\chi_{k n 0}^{(1)}(r)$ being both positive-valued and strictly increasing with $r$ as long as $\chi_{k n 0}(r) \geq 0$. As vanishing of $\chi_{k n 0}\left(r_{0}\right)$ at some $r_{0}>0$ [note that $\chi_{k n 0}(0)=0$ per the boundary conditions (17)] would contradict these findings, the corresponding NO must be nodeless and the moments $\left\{\mu_{k n 0, j}\right\}$ must be positive-valued for all $j$.

For $\omega=\omega_{1}$, the boundary conditions (18) become

$\chi_{1 n 0}^{(1)}(0)=\lambda_{1 n 0}^{-1}\left(C_{10} \mu_{1 n 0,1}+C_{11} \mu_{1 n 0,2}\right)$

and

$\chi_{1 n 0}^{(3)}(0)=2 \lambda_{1 n 0}^{-1} C_{11} \mu_{1 n 0,0}$.

Since both the coefficients $C_{10}$ and $C_{11}$ are positive-valued, one infers from Eq. (26) that at least one of the moments that enters its r.h.s. has to have the same sign as $\lambda_{1 n 0}$. Consequently, all the NOs pertaining to negative-valued natural amplitudes must possess nodes and thus, per the considerations of the preceding paragraph and the boundary condition (27), the corresponding third-order derivatives $\left\{\chi_{k n 0}^{(3)}(0)\right\}$ must be negative-valued and the moments $\left\{\mu_{1 n 0,0}\right\}$ must be greater than zero. On the other hand, nothing precludes the existence of a nodeless NO pertaining to a positive-valued natural amplitude. However, due to the orthonormality of the NOs, only one such natural orbital [characterized by $\left.\chi_{k n 0}^{(3)}(0)>0\right]$ is possible.

All of those inferences carry over to the case of $\omega=\omega_{2}$, where the boundary conditions (26) and (27) are replaced with

$\chi_{2 n 0}^{(1)}(0)=\lambda_{2 n 0}^{-1}\left(C_{20} \mu_{2 n 0,1}+C_{21} \mu_{2 n 0,2}+C_{22} \mu_{2 n 0,3}\right)$

and

$\chi_{2 n 0}^{(3)}(0)=2 \lambda_{2 n 0}^{-1}\left(C_{21} \mu_{2 n 0,0}+3 C_{22} \mu_{2 n 0,1}\right)$, 
the coefficients $C_{20}, C_{21}$, and $C_{22}$ again being all positive-valued.

\subsection{Approximate solutions of Eq. (16)}

For negative-valued natural amplitudes, applying a WKBlike approximation [32] to Eq. (16) yields the (unnormalized) eigenfunctions very close to $n+\frac{1}{4}(n=1,2, \ldots)$. When substituted into Eq. (32), these solutions produce the coefficients $\left\{A_{k n 0}\right\}$ whose rapid decay with $n$ follows the asymptotics of $(-1)^{n+1} \sqrt{2} \exp \left[-\pi\left(n+\frac{1}{4}\right)\right]$. In light of these facts, one expects the quantity $\Delta_{k n 0}$ in the expression

$\chi_{k n 0}(r) \approx\left(\frac{4 \omega_{k}}{\pi}\right)^{1 / 4} \exp \left(\frac{3 \omega_{k}}{8} r^{2}\right)\left\{\sin \left[\pi \beta_{k n 0} \operatorname{erf}\left(\frac{\sqrt{\omega_{k}}}{2} r\right)\right]+A_{k n 0} \sinh \left[\pi \beta_{k n 0} \operatorname{erf}\left(\frac{\sqrt{\omega_{k}}}{2} r\right)\right]\right\}$,

where

$\beta_{k n 0}=\left(-\frac{8 C_{k 1}}{\pi \omega_{k}^{2} \lambda_{k n 0}}\right)^{1 / 4}$

[note that the other two linearly independent approximate solutions that involve the cos and cosh functions do not enter Eq. (30) because of the vanishing of $\chi_{k n 0}(0)$ and $\chi_{k n 0}^{(2)}(0)$ required by the boundary conditions (17)]. The small- $r$ approximation (30) is asymptotically exact at the $\lambda_{k n 0} \rightarrow 0$ limit and is expected to be accurate for $r$ satisfying the inequality $\exp \left(\omega_{k} r^{2}\right) \ll \beta_{k n 0}^{4}$.

In principle, an approximation valid throughout the entire range of radial distances could be obtained by stitching the above result with the large- $r$ asymptotics given by Eq. (20). However, the resulting quantization of the natural amplitudes turns out to be unduly sensitive to the choice of the stitching point, rendering such an approach impractical. Instead, one can either set $A_{k n 0}$ to zero or determine it by matching the radial location of the outermost node of the approximant (30) with that of its exact counterpart. When employed in conjunction with exact natural amplitudes, either of those choices produced NOs of remarkable accuracy (see the next section of this paper).

The large- $r$ asymptotics of the approximant (30) is not compatible with the polynomial (20) unless the latter is set to zero. In that case, one obtains

$A_{k n 0}=-\frac{\sin \pi \beta_{k n 0}}{\sinh \pi \beta_{k n 0}}$

and

$\frac{\tan \pi \beta_{k n 0}}{\tanh \pi \beta_{k n 0}}=1$,

i.e., an approximate quantization of the negative-valued natural amplitudes $\left\{\lambda_{k n 0}\right\}$. Equation (33) admits infinitely many positive-valued solutions, all of which are

$$
\begin{aligned}
\lambda_{k n 0} & =-\frac{8 C_{k 1}}{\pi \omega_{k}^{2}}\left(n+\Delta_{k n 0}\right)^{-4} \\
& =-\frac{4 \Psi\left(\omega_{k} ; \overrightarrow{0}, \overrightarrow{0}\right)}{\pi \omega_{k}^{2}}\left(n+\Delta_{k n 0}\right)^{-4}
\end{aligned}
$$

to be small and dependent on $n$ only weakly.

Setting $A_{k n 0}=0$ in Eq. (30), which implies setting $\Delta_{k n 0}=1$ in Eq. (34) [required by suppression of the leading divergence of $\chi_{n 0}\left(\omega_{k} ; r\right)$ at $r \rightarrow \infty$ ], affords a primitive approximation,

$$
\begin{aligned}
\phi_{n 0}\left(\omega_{k} ; r\right) \approx\left(\frac{4 \omega_{k}}{\pi}\right)^{1 / 4} \exp \left(-\frac{\omega_{k}}{8} r^{2}\right) r^{-1} \\
\times \sin \left[\pi(n+1) \operatorname{erf}\left(\frac{\sqrt{\omega_{k}}}{2} r\right)\right],
\end{aligned}
$$

to the radial part $\phi_{n 0}\left(\omega_{k} ; r\right)$ of the natural orbital pertaining to the natural amplitude $\lambda_{k n 0}$ (note the presence of $n$ radial nodes). Although the approximants (35) are rather inaccurate, for a given $\omega_{k}$ they form a set of orthonormal functions whose simplicity facilitates evaluation of asymptotic expressions for matrix elements of one-electron operators. In particular, one obtains

$$
\begin{aligned}
t_{k n 0} & \equiv t_{n 0}\left(\omega_{k}\right) \\
& =\left\langle\psi_{n 00}\left(\omega_{k} ; \vec{r}\right)\left|-\frac{1}{2} \hat{\nabla}^{2}\right| \psi_{n 00}\left(\omega_{k} ; \vec{r}\right)\right\rangle \underset{n \rightarrow \infty}{\longrightarrow} \frac{\sqrt{3}}{6} \pi \omega_{k} n^{2}
\end{aligned}
$$

and

$$
\begin{aligned}
v_{k n 0} & \equiv v_{n 0}\left(\omega_{k}\right) \\
& =\left\langle\psi_{n 00}\left(\omega_{k} ; \vec{r}\right)\left|\frac{1}{2} \omega_{k}^{2} r^{2}\right| \psi_{n 00}\left(\omega_{k} ; \vec{r}\right)\right\rangle \underset{n \rightarrow \infty}{\longrightarrow} \omega_{k} .
\end{aligned}
$$

In turn, combining Eqs. (34), (36), and (37) with the alternative expression for the natural amplitudes, which reads [28] 
$\lambda_{k n 0}=\frac{\eta_{k n 0}}{E_{k}-2 h_{k n 0}}$,

where

$\eta_{k n 0} \equiv \eta_{n 0}\left(\omega_{k}\right)=\left\langle\psi_{n 00}\left(\omega_{k} ; \vec{r}_{1}\right)\left|\frac{\Psi\left(\omega_{k} ; \vec{r}_{1}, \vec{r}_{2}\right)}{r_{12}}\right| \psi_{n 00}\left(\omega_{k} ; \vec{r}_{2}\right)\right\rangle$,

$E_{k} \equiv E\left(\omega_{k}\right)=\left\langle\Psi\left(\omega_{k} ; \vec{r}_{1}, \vec{r}_{2}\right)|\hat{H}| \Psi\left(\omega_{k} ; \vec{r}_{1}, \vec{r}_{2}\right)\right\rangle, \quad$ and $\quad h_{k n 0} \equiv$ $h_{n 0}\left(\omega_{k}\right)=t_{n 0}\left(\omega_{k}\right)+v_{n 0}\left(\omega_{k}\right)$, produces the asymptotics

$\eta_{k n 0} \underset{n \rightarrow \infty}{\longrightarrow} \frac{8 C_{k 1}}{\sqrt{3} \omega_{k}} n^{-2}$
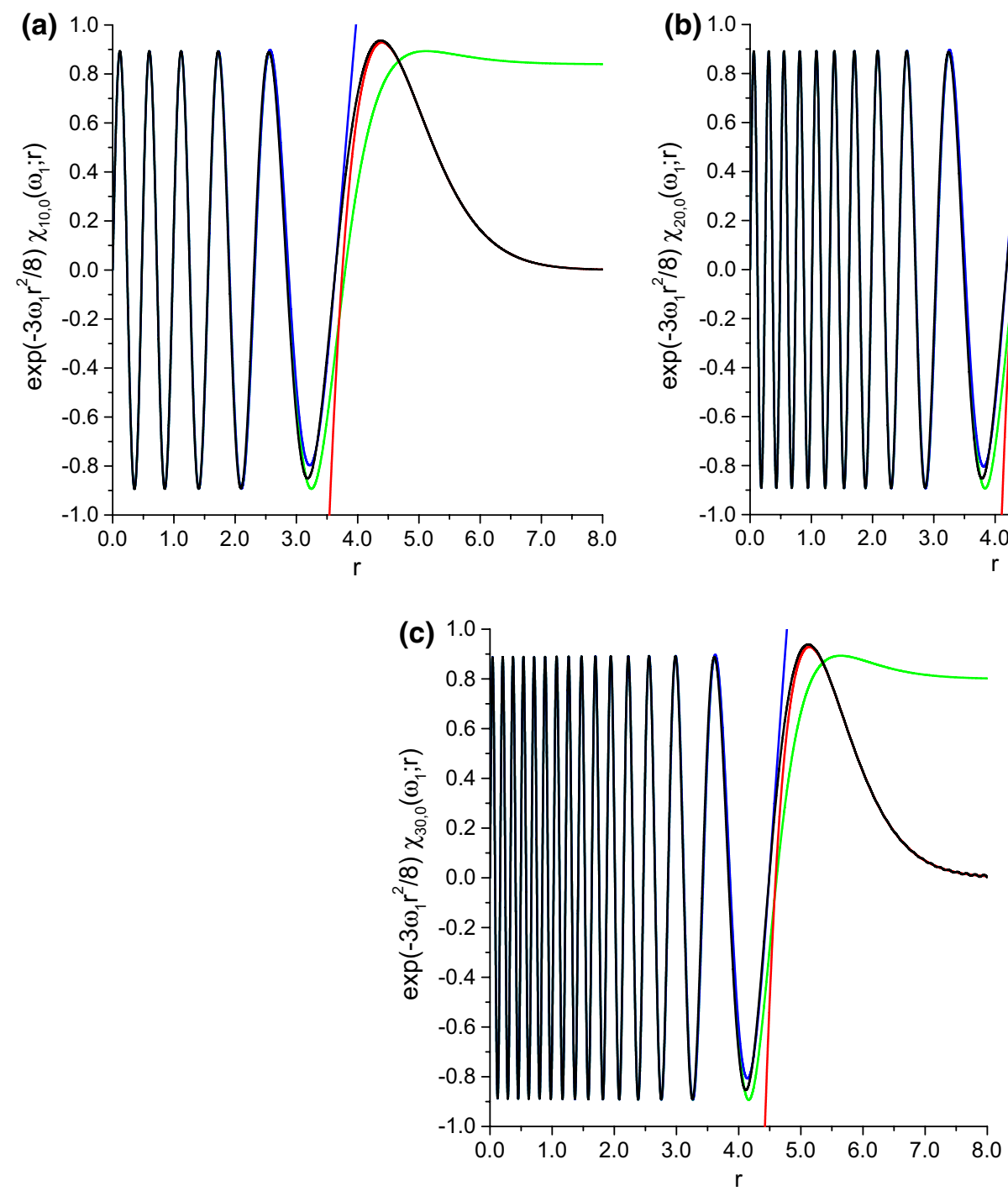

Fig. 1 The exact (black) reduced radial parts $\exp \left(-\frac{3 \omega_{k}}{8} r^{2}\right) \chi_{n 0}\left(\omega_{k} ; r\right)$ of the $s$-type NOs for $k=1$ together with the respective large- $r$ asymptotics (20) (red) and the WKB approximants (30) with $A_{k n 0}$

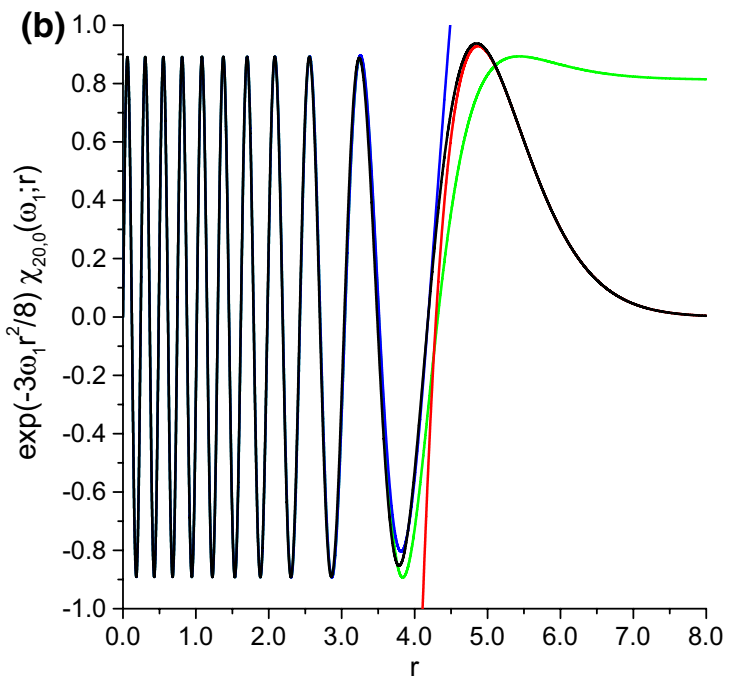

\section{Numerical verification of theoretical predictions}

Numerical algorithms for computation of highly accurate energies $E(\omega)$ and wavefunctions $\Psi\left(\omega ; \vec{r}_{1}, \vec{r}_{2}\right)$ together with the corresponding natural amplitudes $\left\{\lambda_{n l}(\omega)\right\}$ and the spatial parts $\left\{\psi_{n l m}(\omega ; \vec{r})\right\}$ of the natural orbitals for arbitrary finite values of the confinement strength $\omega$ have been described in detail elsewhere [28]. At the $\omega \rightarrow \infty$ limit, these algorithms are superseded by a simplified treatment based upon perturbation theory [25] that involves evaluation of the elements

either set to zero (green) or obtained by matching the radial position of the outermost node (blue): (a) $n=10$, (b) $n=20$, and (c) $n=30$ 

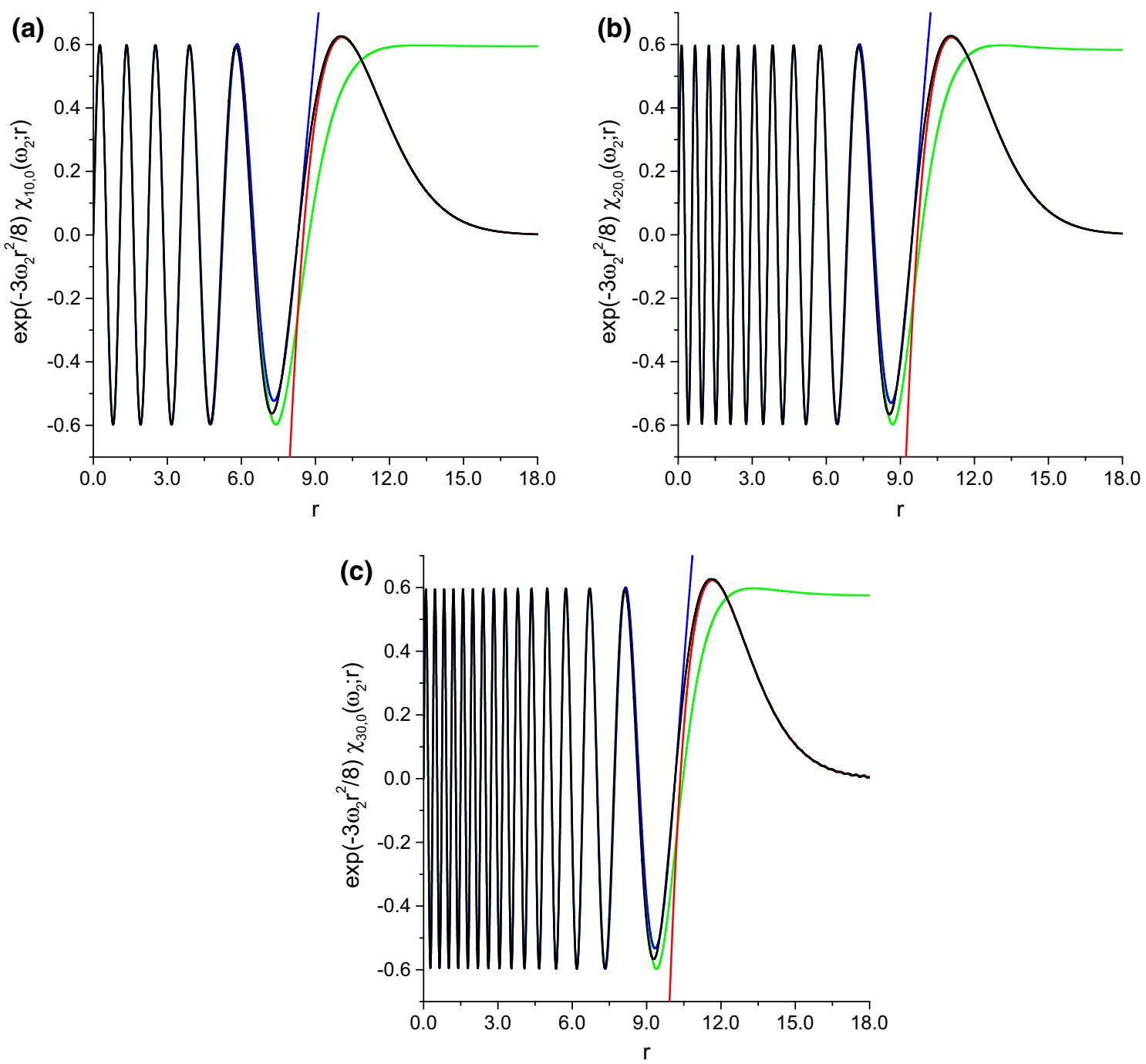

Fig. 2 The exact (black) reduced radial parts $\exp \left(-\frac{3 \omega_{k}}{8} r^{2}\right) \chi_{n 0}\left(\omega_{k} ; r\right)$ of the $s$-type NOs for $k=2$ together with the respective large- $r$ asymptotics (20) (red) and the WKB approximants (30) with $A_{k n 0}$

$$
\begin{aligned}
G_{l, p q} & =-\left(\frac{2}{\pi}\right)^{1 / 2} 2^{4-(2 p+2 q+l)} \frac{(2 p+2 q+2 l-5) !}{(p+q+l-2) !} \\
\times & {\left[\frac{(p+l-1) !(q+l-1) !}{(p-1) !(2 p+2 l-1) !(q-1) !(2 q+2 l-1) !}\right]^{1 / 2} }
\end{aligned}
$$

of the matrices $\left\{\boldsymbol{G}_{l}\right\} \equiv\left\{\left\{G_{l, p q}\right\}_{p, q=1+\delta_{l 0}}^{M}\right\}$ whose eigenvalues approximate the negative-valued reduced natural amplitudes $\left\{\bar{\lambda}_{n l}\right\}$,

$\bar{\lambda}_{n l}=\lim _{\omega \rightarrow \infty} \omega^{1 / 2} \lambda_{n l}(\omega)$, either set to zero (green) or obtained by matching the radial position of the outermost node (blue): (a) $n=10$, (b) $n=20$, and (c) $n=30$

and the respective eigenvectors $\left\{\boldsymbol{D}_{n l}\right\} \equiv\left\{\left\{D_{n l, q}\right\}_{q=1+\delta_{l 0}}^{M}\right\}$ enter the approximants [where $L_{q-1}^{l+1 / 2}(t)$ is the pertinent associated Laguerre polynomial]

$$
\begin{aligned}
& \bar{\psi}_{n l m}(\vec{r}) \approx 2 \pi^{-1 / 4} \sum_{q=1+\delta_{l 0}}^{M}\left[\frac{(2 q-2) ! !}{(2 q+2 l-1) ! !}\right]^{1 / 2} D_{n l, q} \\
& \times L_{q-1}^{l+1 / 2}\left(r^{2}\right)\left(2 r^{2}\right)^{l / 2} \exp \left(-\frac{1}{2} r^{2}\right) Y_{l}^{m}(\theta, \varphi)
\end{aligned}
$$

for the reduced spatial parts $\left\{\bar{\psi}_{n l m}(\vec{r})\right\}$ of the natural orbitals defined as 

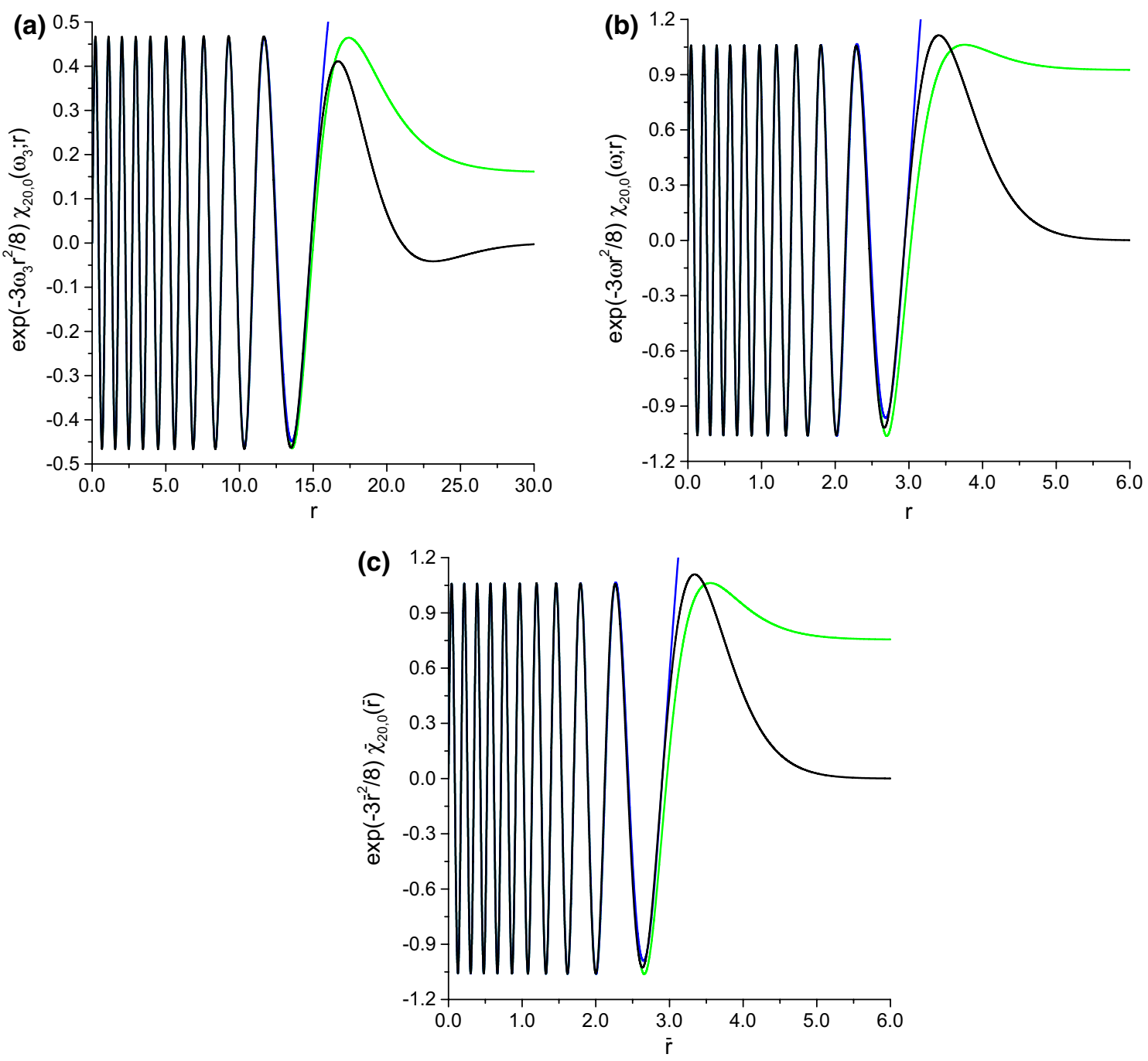

Fig. 3 The exact (black) reduced radial parts $\exp \left(-\frac{3 \omega}{8} r^{2}\right) \chi_{n 0}(\omega ; r)$ of the $s$-type NOs for $n=20$ together with the respective WKB approximants (30) with $A_{k n 0}$ either set to zero (green) or obtained by match-

$\bar{\psi}_{n l m}(\vec{r})=\lim _{\omega \rightarrow \infty} \omega^{-3 / 4} \psi_{n l m}\left(\omega ; \omega^{-1 / 2} \vec{r}\right)$.

Computations of $\operatorname{Tr}\left[\boldsymbol{G}_{1}(\omega)\right]^{2}$ and $\operatorname{Tr}\left[\boldsymbol{G}_{2}(\omega)\right]^{2}$ by infinite algebraic summations yield the large- $\omega$ asymptotics of the collective occupancies per spin and single value of $m$ that read, respectively, $\frac{127-48 \pi+36 \ln 2}{72 \pi} \omega^{-1} \approx 5.11440 \cdot 10^{-3} \omega^{-1}$ and $\frac{-2053+720 \pi-300 \ln 2}{1800 \pi} \omega^{-1} \approx 1.77291 \cdot 10^{-4} \omega^{-1}$, in agreement with the previously published estimates [17]. The numerical data of the present study that are quoted below have been calculated with 2000-digit arithmetic available within the algebraic manipulation software [33] from matrices truncated at $M=1000$.

Numerical verification of the theoretical predictions formulated in the previous section of this paper commences ing the radial position of the outermost node (blue): (a) $\omega=\omega_{3}$, (b) $\omega=1$, and (c) $\omega \rightarrow \infty$ (see the text for explanation)

with comparisons between the exact and approximate natural orbitals (here and in the following, the $s$-type NOs are ordered according to decreasing absolute magnitudes of the corresponding negative-valued natural amplitudes, with $n=1$ assigned to first such NO, etc.). Inspection of Figs. 1 and 2 reveals remarkable accuracy of the reduced radial parts [given by $\exp \left(-\frac{3 \omega}{8} r^{2}\right) \chi_{n 0}(\omega ; r)$ or, equivalently, $\left.\exp \left(\frac{\omega}{8} r^{2}\right) r \phi_{n 0}(\omega ; r)\right]$ of the 10th, 20th, and 30th NOs computed from the approximants (30) at both $\omega=\frac{1}{2}$ and $\omega=\frac{1}{10}$. The excellent agreement at sufficiently small radial distances is unaffected by the choice of $A_{k n 0}$ being either set to zero or determined by matching the radial location of the outermost node of the approximant with that of its exact counterpart. On the other hand, as expected, neither choice gives rise to correct asymptotics at $r \rightarrow \infty$ [which is, however, faithfully 


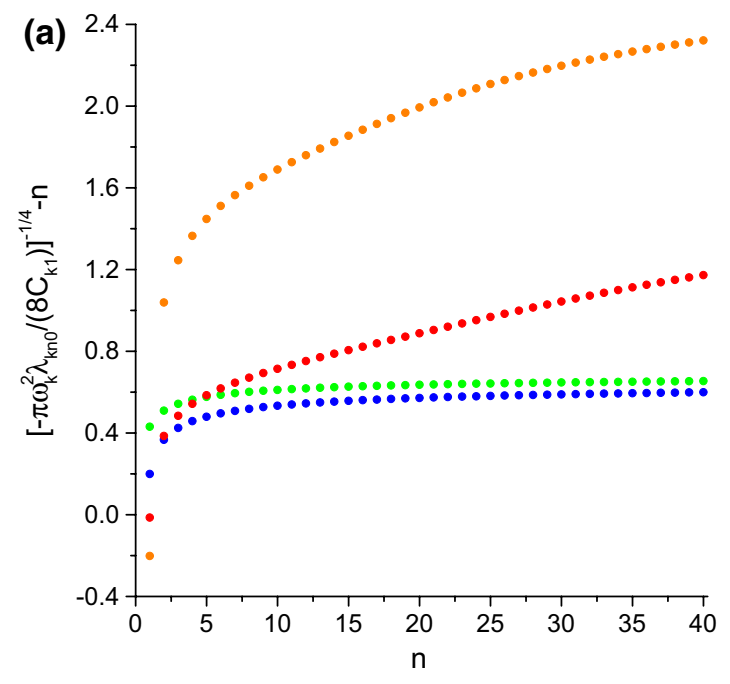

Fig. 4 Deviations from the large- $n$ asymptotics of the natural amplitudes: (a) $\lambda_{k n 0}$ for $k=1$ (green), $k=2$ (blue), $k=3$ (red), and $k=4$

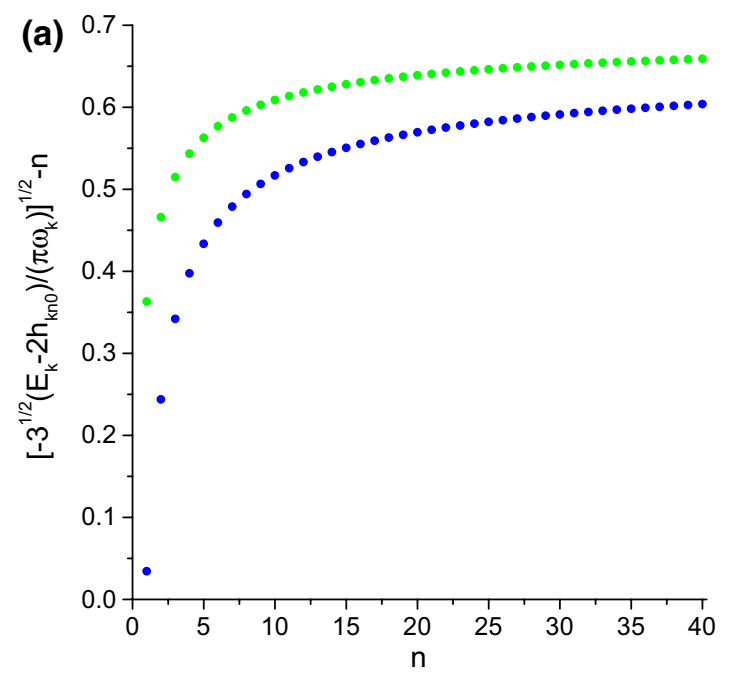

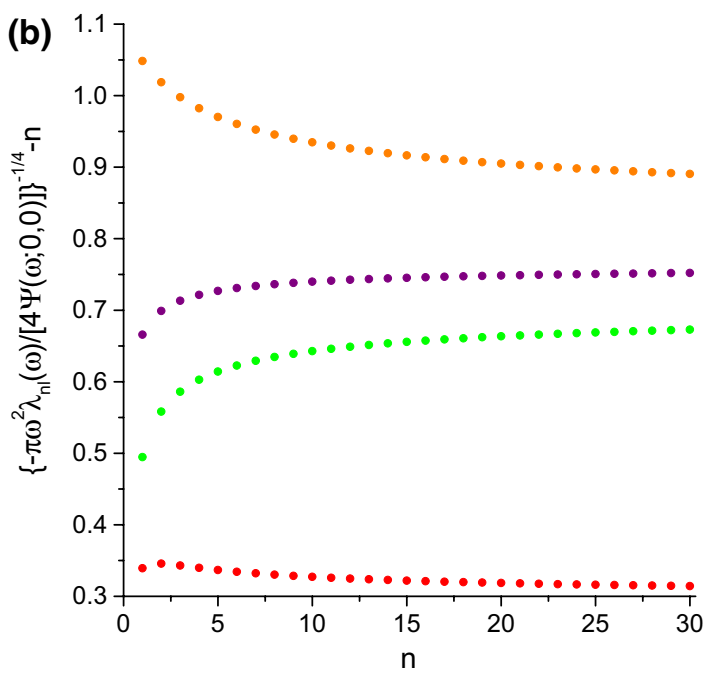

(orange); (b) $\lambda_{n l}$ ( $\left.\omega\right)$ for $\omega=1, l=0$ (green), $\omega \rightarrow \infty, l=0$ (purple), $\omega \rightarrow \infty, l=1$ (red), and $\omega \rightarrow \infty, l=2$ (orange)

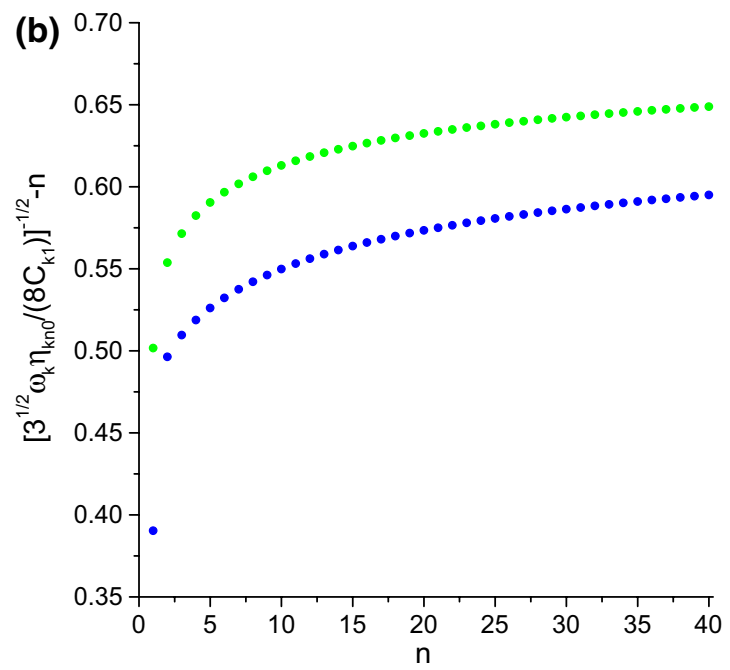

Fig. 5 Deviations from the large- $n$ asymptotics of: (a) the denominators $E_{k}-2 h_{k n 0}$ and (b) the numerators $\eta_{k n 0}$ for $k=1$ (green), $k=2$ (blue), $k=3$ (red), and $k=4$ (orange)

reproduced by $\left.\exp \left(-\frac{3 \omega}{8} r^{2}\right) \chi_{k n 0}^{>}(r)\right]$. These features of the approximants are found to carry over not only to other values of $k$, such as in the case of $\omega=\omega_{3}$ (Fig. 3a), but also to arbitrary magnitudes of the confinement strength such as $\omega=1$ (Fig. 3b) and even the $\omega \rightarrow \infty$ limit of vanishing correlation [Fig. 3c, note that $\bar{\chi}_{n 0}(\bar{r})=\lim _{\omega \rightarrow \infty} \omega^{-1 / 4} \chi_{n 0}\left(\omega ; \omega^{-1 / 2} \bar{r}\right)$ ], uncovering approximate universal scaling of the $s$-type NOs.

Two remarks are in order at this point. First of all, due to the presence of the factor of $\exp \left(\frac{\omega}{8} r^{2}\right)$, the absolute errors exhibited at larger values of $r$ by the approximate reduced radial parts of the NOs displayed in Figs. 1, 2, and 3 are much larger than those of the actual natural orbitals. Second, as the number of nodes increases with $n$, the spacing between them becomes proportionally reduced, leaving the radial extents of the NOs barely changed. This observation explains the large- $n$ asymptotic behavior of the expectation values of both the kinetic energy operator [Eq. (36)] and the operator describing the interaction with the confining potential [Eq. (37)]. The asymptotic constancy of $v_{n 0}(\omega)$ with respect to $n$, which reflects the almost constant radial extents of the NOs, is consistent with the quadratic dependence of $t_{n 0}(\omega)$ on $n$ that is reminiscent of that known for a particle in one-dimensional box. These asymptotics are 
expected to persist for all values of $\omega$, including the limit of $\omega \rightarrow \infty$ at which the interelectron interaction becomes vanishingly small in comparison with the other components of the Hamiltonian (1). When juxtaposed against the linear dependences on $n$ exhibited by the kinetic and potential energy components pertaining to the $s$-type wavefunctions of the three-dimensional harmonic oscillator (which become more and more diffuse upon successive excitations), these asymptotics vividly illustrate the fundamental difference between the natural orbitals and the eigenfunctions of the one-electron Hamiltonians that are left in $\hat{H}$ upon removal of the interelectron interaction term.

The natural amplitudes $\left\{\lambda_{k n 0}\right\}$ computed for $k=1, k=2$, $k=3$, and $k=4$ are found to follow the asymptotics (34) as attested by both the smallness and the weak dependence on $n$ of the expression $\left(-\frac{\pi \omega_{k}^{2} \lambda_{k n 0}}{8 C_{k 1}}\right)^{-1 / 4}-n$ (Fig. 4a). In general, the rate at which this asymptotics is approached appears to diminish with increasing $k$. Quite unexpectedly, the natural amplitudes $\left\{\lambda_{n l}(\omega)\right\}$ computed at arbitrary values of $\omega$ and even for $l \neq 0$ turn out to exhibit the same large- $n$ behavior (which is reflected in the values of $\left[-\frac{\pi \omega^{2} \lambda_{n l}(\omega)}{4 \Psi(\omega ; \overrightarrow{0}, \overrightarrow{0})}\right]^{-1 / 4}-n$ plot-

ted against $n$ in Fig. 4b). The analogous plots of the quantities $\left[-\frac{\sqrt{3}\left(E_{k}-2 h_{k n 0}\right)}{\pi \omega_{k}}\right]^{1 / 2}-n$ (Fig. 5a) and $\left(\frac{\sqrt{3} \omega_{k} \eta_{k n 0}}{8 C_{k 1}}\right)^{-1 / 2}-n$

(Fig. 5b) confirm the predicted asymptotics (36), (37), and (40).

\section{Conclusions}

The radial components of the natural orbitals (NOs) pertaining to the ${ }^{1} S_{+}$ground state of the two-electron harmonium atom are found to satisfy homogeneous differential equations at the values of the confinement strength $\omega$ at which the respective correlation factors are given by polynomials. Together with the angular momentum $l$ of the NOs, the degrees of these polynomials determine the orders of the differential equations, eigenvalues of which (arising from welldefined boundary conditions) yield the natural amplitudes. In the case of $l=0$, analysis of these equations uncovers certain properties of the NOs whereas application of a WKBlike approximation produces asymptotic expressions for both the NOs and the corresponding natural amplitudes that hold when the latter are small negative numbers. Extensive numerical calculations reveal that these expressions remain valid for arbitrary values of $\omega$. The approximate $s$-type NOs, which are remarkably accurate at sufficiently small radial distances and exhibit universal scaling, differ qualitatively from the eigenfunctions of the core Hamiltonian even at the $\omega \rightarrow \infty$ limit of vanishing electron correlation.
The prediction that the product of the $n$th negative-valued natural amplitude and $n^{4}$ tends to a constant at the $n \rightarrow \infty$ limit implies the asymptotic $n^{-8}$ decay of the occupation numbers $\left\{v_{n l}(\omega)\right\}$ of the NOs. In fact, the expression

$\nu_{n l}(\omega)=\frac{16|\Psi(\omega ; \overrightarrow{0}, \overrightarrow{0})|^{2}}{\pi^{2} \omega^{4}}\left[n+\Delta_{n l}(\omega)\right]^{-8}$,

where $\left\{\Delta_{n l}(\omega)\right\}$ are small numbers weakly dependent on $n$, appears to be universal, i.e., to hold for arbitrary $\omega$ and $l$. Interestingly, naive summation of this expression over $n$ yields collective occupancies that exhibit the same proportionality constant of $\frac{|\Psi(\omega ; \overrightarrow{0}, \overrightarrow{0})|^{2}}{\omega^{4}}$ as those that follow from the large-l asymptotics of Hill [34].

Acknowledgements The research described in this publication has been funded by the National Science Center (Poland) under Grant 2016/21/B/ST4/00597. Support from MPI PKS Dresden is also acknowledged.

Open Access This article is distributed under the terms of the Creative Commons Attribution 4.0 International License (http://creativeco mmons.org/licenses/by/4.0/), which permits unrestricted use, distribution, and reproduction in any medium, provided you give appropriate credit to the original author(s) and the source, provide a link to the Creative Commons license, and indicate if changes were made.

\section{References}

1. Taut M (1993) Phys Rev A 48:3561

2. Sahni V (2010) Quantal density functional theory II: approximation methods and applications. Springer, Berlin

3. Gori-Giorgi P, Savin A (2009) Int J Quantum Chem 109:2410

4. Zhu WM, Trickey SB (2006) J Chem Phys 125:094317

5. Hessler P, Park J, Burke K (1999) Phys Rev Lett 82:378

6. Ivanov S, Burke K, Levy M (1999) J Chem Phys 110:10262

7. Qian Z, Sahni V (1998) Phys Rev A 57:2527

8. Taut M, Ernst A, Eschrig H (1998) J Phys B 31:2689

9. Huang CJ, Umrigar CJ (1997) Phys Rev A 56:290

10. Filippi C, Umrigar CJ, Taut M (1994) J Chem Phys 100:1290

11. Kais S, Hersbach DR, Handy NC, Murray CW, Laming GJ (1993) J Chem Phys 99:417

12. Laufer PM, Krieger JB (1986) Phys Rev A 33:1480

13. Elward JM, Hoffman J, Chakraborty A (2012) Chem Phys Lett 535:182

14. Elward JM, Thallinger B, Chakraborty A (2012) J Chem Phys 136:124105

15. Glover WJ, Larsen RE, Schwartz BJ (2010) J Chem Phys 132:144101

16. Rodríguez-Mayorga M, Ramos-Cordoba E, Via-Nadal M, Piris M, Matito E (2017) Phys Chem Chem Phys 19:24029

17. Cioslowski J, Pernal K (2000) J Chem Phys 113:8434 (and the references cited therein)

18. White RJ, Byers Brown W (1970) J Chem Phys 53:3869

19. Benson JM, Byers Brown W (1970) J Chem Phys 53:3880

20. Cioslowski J (2013) J Chem Phys 139:224108

21. Cioslowski J, Matito E (2011) J Chem Phys 134:116101

22. Gill PMW, O'Neill DP (2005) J Chem Phys 122:094110

23. Cioslowski J, Buchowiecki M (2006) J Chem Phys 125:064105 
24. Cioslowski J, Buchowiecki M (2005) J Chem Phys 122:084102

25. Cioslowski J (2015) Theor Chem Acc 134:113

26. King HF (1996) Theor Chim Acta 94:345

27. Cioslowski J, Buchowiecki M (2005) J Chem Phys 123:234102

28. Cioslowski J (2018) J Chem Phys 148:134120

29. Giesbertz KJH, van Leeuwen R (2013) J Chem Phys 139:104109

30. Giesbertz KJH, van Leeuwen R (2013) J Chem Phys 139:104110
31. Löwdin P-O, Shull H (1956) Phys Rev 101:1730

32. Zwillinger D (1997) Handbook of differential equations. Academic Press, New York

33. Mathematica Version 9.0 (2013) Wolfram Research Inc. Champaign, IL

34. Hill RN (1985) J Chem Phys 83:1173 